\title{
Genetic mapping of QTLs controlling the ISIAH hypertensive rat behavior in an open field tests
}

\author{
Olga Redina \\ Laboratory of Evolutional Genetics, \\ Institute of Cytology and Genetics, \\ Siberian Branch of Russian Academy \\ of Sciences \\ Novosibirsk, Russia \\ oredina@bionet.nsc.ru
}

\author{
Svetlana Smolenskaya \\ Laboratory of Evolutional Genetics, \\ Wheat Genetics Laboratory, \\ Institute of Cytology and Genetics, \\ Siberian Branch of Russian Academy \\ of Sciences \\ Novosibirsk, Russia \\ svsmol@ngs.ru
}

\author{
Arcady Markel \\ Laboratory of Evolutional Genetics, \\ Institute of Cytology and Genetics, \\ Siberian Branch of Russian Academy \\ of Sciences \\ Novosibirsk, Russia \\ markel@bionet.nsc.ru
}

\begin{abstract}
Genetic mapping of QTLs controlling the ISIAH hypertensive rat behavior in an open field tests evaluating research behavior, the level of anxiety and motor activity of animals was performed. The work was carried out using two groups of male hybrid rats F2(ISIAHxWAG) of different ages 3-4 months $(n=103)$ and 6 months $(n=126)$ obtained by crossing hypertensive ISIAH and normotensive WAG rats whose behaviors are significantly different in all six tests in an open field taken in the analysis: the latency, the motor activity at 1-st minute of the test, motor activity on the periphery, grooming on the periphery, rearing on the periphery, defecation score. In both groups of rats, genetic loci associated with the studied traits of behavior were found and the common loci associated with both the behavioral traits and traits related to the hypertension development were identified. The results of our work suggest that genes that control behavioral patterns of ISIAH rats can either be closely linked to genes involved in the control of traits associated with the hypertensive status of these rats, or, in some loci, may have a pleiotropic effect on both behavioral traits and on the traits associated with the hypertensive status of ISIAH rats.
\end{abstract}

Keywords - QTL analysis, behavior, open field tests, hypertensive ISIAH rats

\section{Introduction}

The increased stress sensitivity of the mammalian organism is associated with its special neuroendocrine status, which affects behavior, including motor activity, anxiety, changes in cognitive ability, and many other physiological functions, in particular, the impaired control of the blood pressure and development of hypertension [1-3]. The ISIAH rats simulating a stress-sensitive form of arterial hypertension are characterized by increased activity of the hypothalamicpituitary-adrenal and sympathetic adrenal systems, changes in the functions and morphology of a number of peripheral target organs (brain, kidneys, adrenal glands, heart), as well as specific behavior in unfamiliar environments [4-6]. The literature suggests that changes in behavior may be a condition concomitant to hypertension. However, the genetic basis of the relationship between behavior and the body's hypertensive status have been poorly understood to date, and such studies have not been conducted on ISIAH rats before.

The aims of current work were 1) to perform genetic mapping (QTL analysis) of ISIAH rat behavior in six openfield tests evaluating research behavior, the level of anxiety and motor activity of animals, and 2) to identify the common loci associated with both the behavioral traits and traits related to the development of hypertension.
Methods

Animals

Two groups of male hybrid rats F $_{2}$ (ISIAHxWAG) of different ages - 3-4 months $(n=103)$ and 6 months $(n=126)$ obtained by crossing rats of the hypertensive ISIAH strain and the normotensive WAG strain were used. The behavior of rats from these two strains is significantly different in all six tests in an open field taken in the analysis: the latency, the motor activity at 1-st minute of the test, motor activity on the periphery, grooming on the periphery, rearing on the periphery, defecation score.

\section{QTL analysis}

QTL analysis was performed using 145 polymorphic microsatellite markers in the group of younger rats, and using 149 polymorphic microsatellite markers in the group of older rats. The list of DNA markers and the sequence of primers used are given on the website of the Institute of Cytology and Genetics SB RAS (http://icg.nsc.ru/isiah/en/category/qtl/). Processing the results was carried out using specialized programs MAPMAKER/EXP3.0 and MAPMAKER/QTL1.1. Identification of common loci associated both with the behavior of rats and with other traits associated with the hypertensive phenotype of the ISIAH rats, such as blood pressure levels at rest and under stress, body weight, absolute and relative weights of target organs (kidneys, adrenal glands, heart), the concentration of corticosterone in the plasma at rest and under stress, and the concentration of norepinephrine in the hypothalamus was performed using a bivariate (multivariate) analysis in the QTL Cartographer Version 1.17 software package, JZmapqtl (statgen.ncsu.edu). Empirical significant threshold values for QTLs were determined by a permutation test using 1000 permutations of the original data.

\section{Results}

In both groups of rats, genetic loci associated with the studied traits of behavior were found. In the group of rats aged 3-4 months, statistically significant associations were found for such traits as motor activity at the 1st minute of the test at the locus on chromosome 16 (marker D16Rat58), motor activity at the periphery at the loci on chromosomes 2 and 16 (markers D2Rat157 and D16Rat32*), grooming at the periphery on chromosome 20 (marker D20Rat30) and latent period on chromosome 11 (marker D11Rat50*). In the group of rats at the age of 6 months, the most highly reliable associations were found for defecation on chromosomes 2 and 14 (markers D2Rat194* and D14Rat5*). Asterisks mark loci whose association with the trait is described for the first time. 
A search for common loci showed that some loci associated with behavior overlap with loci associated with one or more of the analyzed traits characterizing the hypertensive status of ISIAH rats: blood pressure under stress, body weight, relative weight of the adrenal glands, absolute and relative kidney weight, plasma corticosterone concentration at rest and under stress, and the concentration of norepinephrine in the hypothalamus.

\section{Conclusion}

The results of our work suggest that genes that control behavioral patterns of ISIAH rats can either be closely linked to genes involved in the control of traits associated with the hypertensive status of these rats, or, in some loci, may have a pleiotropic effect on both behavior and on the manifestation of traits associated with the hypertensive status of ISIAH rats. The results obtained in this study can be used to determine candidate genes at genetic loci associated with behavioral patterns of hypertensive ISIAH rats.

\section{ACKNOWLEDGMENT}

The work was supported by Russian Foundation for Basic Research grant No. 20-04-00119a and by Budget project No. 0324-2019-0041-C-01.

\section{REFERENCES}

[1] B. Bohus, R. F. Benus, D. S. Fokkema, J. M. Koolhaas, C. Nyakas, G. A. van Oortmerssen, A. J. Prins, A. J. de Ruiter, A. J. Scheurink, and A. B. Steffens, "Neuroendocrine states and behavioral and physiological stress responses," Prog. Brain Res., vol. 72, pp. 57-70, 1987.

[2] F. Kiefer, and K. Wiedemann, "Neuroendocrine pathways of addictive behavior," Addict. Biol., vol. 9, no. 3-4, pp. 205-212, 2004.

[3] R. S. Fries, P. Mahboubi, N. R. Mahapatra, S. K. Mahata, N. J. Schork, G. W. Schmid-Schoenbein, and D. T. O'Connor, "Neuroendocrine transcriptome in genetic hypertension: multiple changes in diverse adrenal physiological systems," Hypertension, vol. 43, no. 6, pp. 13011311, 2004.

[4] I. O. Meshkov, T. A. Alekhina, T. A. Moreva, and A. L. Markel', "[Behavioral characterictics of ISIAH rat strain]," Zh. Vyssh. Nerv. Deiat. Im. I. P. Pavlova, vol. 62, no. 2, pp. 233-242, 2012. Russian.

[5] A. L. Markel, O. E. Redina, M. A. Gilinsky, G. M. Dymshits, E. V. Kalashnikova, Y. V. Khvorostova, L. A. Fedoseeva, and G. S. Jacobson, "Neuroendocrine profiling in inherited stress-induced arterial hypertension rat strain with stress-sensitive arterial hypertension," J. Endocrinol., vol. 195, no. 3, pp. 439-450, 2007.

[6] O.E. Redina, N.A. Machanova, V.M. Efimov, and A.L. Markel. Rats with Inherited Stress-Induced Arterial Hypertension (ISIAH strain) display Specific Quantitative Trait Loci for Blood Pressure and for Body and Kidney Weights on Chromosome 1. Clin. Exp. Pharm. Phys., vol. 33, pp. 456-464, 2006. 